\title{
La importancia de los intereses académicos en la política científica y tecnológica catalana*
}

\author{
Laura Cruz Castro \\ María Fernández Mellizo-Soto \\ Luis Sanz Menéndez \\ Consejo Superior de Investigaciones Científicas (CSIC) \\ Unidad de Políticas Comparadas-Grupo SPRITTE. Madrid
}

\section{Resumen}

Este artículo describe la emergencia y orientación de las políticas de $\mathrm{I}+\mathrm{D}$ e innovación en Cataluña. Se analizan cuáles son los factores más influyentes en la orientación de estas políticas y, en definitiva, en las opciones políticas que se toman. La política de ciencia y tecnología desarrollada por el gobierno regional catalán desde principios de los años ochenta ha sido una política en la que, a pesar de las preferencias manifestadas en el discurso político, ha predominado un modelo de política académico sobre el de orientación empresarial. Asimismo, en términos organizativos e institucionales, en la Administración autonómica, la política científica ha estado separada y diferenciada de la política tecnológica a pesar del diseño inicial de instituciones interdepartamentales.

La principal razón de que la política de $\mathrm{I}+\mathrm{D}$ catalana no siguiera un modelo más industrial, ligado al mundo empresarial, fue la presión que ejercieron las universidades catalanas para que, tanto el diseño institucional como el contenido de la política se adaptara a sus necesidades. La trayectoria académica previa de los gestores también contribuyó a la reorientación de las preferencias políticas. A pesar de la importancia de las empresas catalanas en la $\mathrm{I}+\mathrm{D}$, éstas no se movilizaron ni presionaron a los gobiernos suficientemente. Analíticamente, este caso ilustra cómo la sola creación política de instituciones no garantiza la realización de las preferencias políticas. También pone de manifiesto cómo el horizonte temporal de la toma de decisiones gubernamental tiene un efecto en las expectativas de los actores, que desarrollan procesos de aprendizaje a partir de las experiencias en arenas políticas similares a otros niveles. Por último, destaca la importancia del poder en las instituciones de gestión en este tipo de política distributiva.

Palabras clave: políticas científica y tecnológica, I+D, innovación, intereses, instituciones, Cataluña.

Este trabajo se ha realizado gracias a la financiación del Programa Marco de I+D, del PRICIT de la Comunidad de Madrid y del III Plan Nacional de I+D de la CICYT (SEC 19990829-C02-01).

** Autor de contacto: Luis Sanz Menéndez (lsanz@iesam.csic.es). 
Abstract. The relevance of academic interests in the Catalonian science and technology policy

This paper describes the emergence and orientation of $R \& D$ and innovation policies in Catalonia. The factors with a greater explanatory power about the orientation of these policies are also analised. The research and technology policy developed by the Catalonian government since the beginning of the eighties has been, despite the preferences revealed by the political discourse, one in which an academic model has been predominant over a pro-business one. In the same way, in organisational and institutional terms, research and technology policies have belonged to separate and independent administrative domains, despite the initial creation of inter-departamental institutions within the regional administration.

The main reason why Catalonian R\&D policy has not followed a pro-business model, was the strong pressure that Catalonian Universities showed, in order to get both institutional designs and policy contents which favoured them and which matched their needs. The previous academic background of policy makers contributed in the same direction. In spite of the relevance of firms in the Catalonian R\&D scene, these actors did not either mobilise or pressure governments sufficiently. From an analytical point of view, this case illustrates how the political creation of institutions is not a sufficient condition for the advancement of political preferences. It also makes clear how the time framework of political decision making has an effect on actors' expectations, who develop learning processes from experiences in similar political arenas at different levels. Lastly, it shows how, within these type of distributive policies, decision power within management institutions becomes very important.

Key words: science and technology policy, R\&D, innovation, interests, institutions, Catalonia.

\section{Sumario}
1. Introducción
4. Conclusiones
2. Planteamiento analítico Bibliografía
y marco teórico

3. La emergencia y evolución de la política catalana de ciencia y tecnología

\section{Introducción}

El objeto de este artículo son las políticas de investigación e innovación adoptadas por los gobiernos regionales en Cataluña y los determinantes, de naturaleza política u otra, de las mismas.

El esfuerzo que los países dedican a las actividades de investigación e innovación es un factor decisivo del bienestar de las sociedades; disponer de recursos en investigación y demostrar una fuerte capacidad de innovación tecnológica se reconocen como elementos que condicionan el crecimiento económico de los países (Banco Mundial, 1999).

La teoría económica parece confirmar que los esfuerzos en I+D y en tecnología son determinantes de los niveles de competitividad de las economías 
(Nelson y Romer, 1996) y, además, acepta la existencia de propiedades sistémicas de las economías nacionales que determinarían los resultados económicos: Los sistemas nacionales de innovación (Nelson, 1993; Lundvall, 1992; Freeman, 1995; Edquist, 1997) emergen como categoría que quiere explicar la diversidad de los resultados, en cuanto a crecimiento económico, a partir de las diferencias en los procesos de organización y desarrollo de la innovación.

La retórica de los sistemas de innovación se ha extendido también a ámbitos regionales (Braczyk, Cooke y Heidenreich, 1998; Olazarán y Gómez Uranga, 2001). En general, la aplicación de estos términos se refiere a un subconjunto de actores y actividades de $\mathrm{I}+\mathrm{D}$ o innovación que se localizan en un territorio y que se suelen describir como coherentes desde el punto de vista regional. Sin embargo, estudios empíricos argumentan contundentemente contra la existencia de esos sistemas regionales de innovación (para el caso catalán, véase Riba y Leydesdorff, 2001).

Los análisis realizados sobre los «sistemas regionales de innovación» en España merecen una valoración desigual. Algunos han resaltado las especificidades políticas regionales y han señalado la relevancia de la política de I+D (Cooke, Gómez-Uranga y Etxebarría, 1997; Cooke y Gómez-Uranga, 1998); otros trabajos han regionalizado las políticas nacionales (Durán, 1999) o han descrito las iniciativas regionales (González de la Fé, 2001); por último, el resto presenta un conjunto de datos de la economía regional con atribuciones causales a las políticas regionales no demostradas (para Cataluña, véase, por ejemplo, Bacaría, Borrás y Fernández-Ribas, 2001). Muy pocos han intentado explicar las políticas adoptadas por los gobiernos regionales (para el País Vasco, véase Moso y Olazarán, 2001, y para Cataluña, Vilalta, 2000) y en contadas ocasiones se ha intentado superar el enfoque del caso para dar cuenta de por qué los gobiernos regionales, enfrentados a desafíos similares, con frecuencia, han optado por políticas muy diversas (para Madrid: Sanz Menéndez, Cruz y Romero, 2001; para Galicia: Fernández, Sanz Menéndez y Cruz, 2003; para Andalucía: Romero, Cruz y Sanz Menéndez, 2003).

La relevancia de la política en estos estudios ha sido generalmente escasa, aunque suelen extraer conclusiones de tipo normativo o prescriptivo (Metcalfe y Georghiou, 1998), sin embargo, en éstos y aquellos trabajos, aunque incorporadas las políticas de $\mathrm{I}+\mathrm{D}$ como variables intervinientes e incluso independientes, generalmente se hace muy poca mención a variables explicativas de naturaleza política y están ausentes como variables a ser explicadas ${ }^{1}$.

En este artículo se describen, se analizan y se explican las políticas de ciencia, tecnología e innovación seguidas por los gobiernos catalanes desde 1980 hasta la actualidad. Este estudio se enmarca dentro de unas preguntas de investigación más generales: ¿por qué, y en qué circunstancias, surgen las intervenciones de los gobiernos regionales en materia de $\mathrm{I}+\mathrm{D}$ ?, ¿qué forma adoptan?,

1. Excepciones son, por ejemplo: Ziegler, 1997; Peterson y Sharp, 1998, y Sanz Menéndez, 2001. 
¿cuáles son los factores más influyentes en la forma que esta política adopta y, en definitiva, en las opciones que se toman?, ¿por qué cambian las políticas?

El caso catalán es especialmente interesante por la prioridad política otorgada a la I+D, que hacía que, en 2001, la Generalitat fuera el Gobierno regional de España que, en términos absolutos, más invertía en $\mathrm{I}_{+} \mathrm{D}^{2}$. El caso que presentamos nos permite ver cómo, y en qué circunstancias, las preferencias de los actores políticos cambian y se redefinen, en el propio proceso de construcción de las instituciones responsables de la política. También se observa cómo las batallas en torno al diseño institucional son condicionantes importantes de las opciones de política. Por último, se evidencia que las "preferencias políticas» no son suficientes como factores explicativos del tipo de política que los gobiernos adoptan.

Las políticas adoptadas se redefinen como resultado de la presión institucionalizada de los intereses, a los que se ha dado acceso en las instituciones, especialmente de aquéllos que dependen más de los recursos públicos: los intereses de los académicos. Así, la fuerte presencia institucional de estos intereses hace que se imponga una política regional basada en un modelo o en una forma de interpretar la política de investigación que responde a esos intereses, más allá de los modelos que asocian estas políticas al crecimiento económico y la competitividad. Incluso se observa que, incorporados al sistema institucional, los intereses desarrollan procesos de «aprendizaje político» (policy learning) (Hall, 1993) que permiten la adaptación del discurso político a sus necesidades.

\section{Planteamiento analítico y marco teórico}

Las políticas son un conjunto de actuaciones complejas de los gobiernos en un área o sobre un problema. La definición del problema (Schön y Rein, 1994) y la forma en la que se introduce en la agenda política (Kingdon, 1984) son elementos importantes en la explicación de las opciones tomadas. Además, la acción política tiende a construir un policy domain que tiene asociada una policy community en la que con frecuencia los intereses juegan un papel decisivo (Walker, 1991). Por otro lado, la evolución de los problemas en el mundo real provoca un proceso de transformación de los límites de las políticas (boundaries); la asociación de problemas y la interacción entre ellos en el contexto de la agenda política hace que evolucione el discurso y el juego de la política (Carmines y Stimson, 1989). Por último, cómo está organizado el gobierno y la Administración pública, e institucionalizada la relación con los actores, son también elementos importantes a la hora de entender la dinámica de los grupos que presionan sobre el gobierno (Skocpol y Finegold, 1982).

Lo que tradicionalmente se entiende por "política de ciencia y tecnología» es esencialmente una política de asignación de recursos, una política que se

2. Para una comparación de las políticas de gasto en $\mathrm{I}+\mathrm{D}$ de las regiones, véase la Memoria de Actividades de $I+D+I 2001$ que prepara la Comisión Interministerial de Ciencia y Tecnología (CICYT). 
podría esperar determinase un juego político de naturaleza distributiva (Lowi, 1972), un sistema de reparto de recursos entre los actores del sistema. Estos procesos de distribución exigen mecanismos de «legitimación» que hacen que la relación entre el gobierno, y sus instituciones administrativas, con los clientes sea estrecha; sin embargo, las bases de legitimidad de las políticas que benefician a los académicos son distintas de aquéllas que favorecen a las empresas. En el primer caso, la calidad o la excelencia y la implicación de los interesados — bien personal o institucionalmente- en los sistemas de asesoramiento (qué hay que hacer y a quién hay que dar el dinero para hacerlo) son los mecanismos aceptados; frente a ello, la lógica de las consecuencias, de los efectos de los recursos asignados en el crecimiento económico, está implícita en la orientación de la política a favor de los intereses empresariales. En todo caso, los clientes tienden a construir redes de tipo "mesocorporativista» para la gestión de los recursos.

En este contexto, nuestro análisis pretende caracterizar la variable dependiente, la política de ciencia y tecnología, por su «orientación» — a quien beneficia directamente-. Ésta puede responder, en mayor o menor medida, a dos modelos, que suelen aparecer combinados, pero donde predomina uno sobre el otro: en primer lugar, el modelo académico, caracterizado por dirigirse al fomento de la investigación académica y por ser sus destinatarios principales las universidades y los centros públicos de investigación; en segundo lugar, el modelo empresarial, que concede mayor relevancia a la investigación aplicada y al proceso de innovación tecnológica en las empresas. Así pues, dar respuesta a la vieja pregunta de Lasswell «Who gets what, when and how?» es, en nuestra opinión, el primer paso para caracterizar las políticas y el juego político en torno a ellas.

Las consecuencias de los modelos que se adopten, tanto en términos de «bienestar» como en los efectos esperados sobre el «crecimiento económico», son muy diversas. Las diferentes formas de intervenir tienen diferentes consecuencias y los gobiernos han tendido en los últimos años a redefinir sus políticas de investigación en línea con algunas estimaciones o evidencias sobre los «efectos de las políticas» (Caracostas y Muldur, 1998; EC, 1995). En general, los gobiernos han puesto énfasis creciente en la necesidad de medidas para fomentar las inversiones privadas en investigación, para incentivar a las empresas o para elevar el nivel tecnológico de las mismas. En este nuevo contexto se pretendía que las políticas tradicionales de financiación de la investigación académica, se subordinasen a la transferencia de tecnología, al desarrollo de colaboraciones con la empresa, o a la difusión.

Pues bien, a pesar de que parece que las políticas más idóneas para hacer avanzar el crecimiento económico y la competitividad se relacionan más con el "modelo empresarial», la existencia de una industria potente en la región no es condición suficiente para que los gobiernos adopten políticas de esta orientación, como tampoco los son las preferencias políticas de los gobiernos por sí solas. En general, se constata que a mayor desarrollo de sistema académico (con independencia del nivel de desarrollo del sistema empresarial) más dificultades tiene el gobierno regional para imponer los objetivos (incluso a veces ni se lo plantea) de una política de investigación e innovación (de hecho, el 
cambio de énfasis en el nombre es ya un indicio) para favorecer el crecimiento económico. Además, se observa cómo resulta difícil que los gobiernos, una vez establecidas estructuras institucionales, y pobladas éstas por investigadores académicos, reorienten de forma significativa sus estrategias hacia los intereses empresariales.

La dependencia de los recursos que el gobierno distribuye puede explicar, en parte, la movilización diferencial de los actores que se benefician directamente de los mismos, aunque indirectamente se beneficie también la sociedad. Como la teoría de la acción colectiva predice, los incentivos selectivos son de gran importancia; por otro lado, los actores empresariales que se movilizan en torno al gobierno regional son PYMES que apenas se interesan por este policy domain, mientras que los actores empresariales relevantes en innovación seguramente orientan sus actuaciones, por el volumen de los recursos en juego, al gobierno central o la UE.

La caracterización de la política científica y tecnológica ${ }^{3}$, a lo largo del tiempo, y su clasificación de acuerdo con los dos tipos ideales propuestos, se ha

3. Este trabajo se ha desarrollado empíricamente por medio de métodos cualitativos: análisis documental y entrevistas en profundidad, con un cuestionario semiestructurado de más de una hora de duración, a responsables de la política científica y tecnológica catalana. Se realizaron veinte entrevistas entre mediados de noviembre de 2000 y finales de enero de 2001 a las siguientes personas: Joan Albaigès, delegado del CSIC en Cataluña (1989-1992), director general de Investigación (1993-1995) y comisario de Universidades e Investigación (1995-1999). Miquel Barceló, director del Instituto Catalán de Tecnología (1986-1999) y actualmente parlamentario del PSC en el Parlamento catalán. Artur Bladé, jefe del Gabinete técnico de investigación de la Dirección General de Universidades de la GC (1982-1988), secretario general de la CIRIT (1988-1992), subdirector general de Investigación del Departamento de Educación (1992-2000) y asesor de Relaciones Internacionales del consejero del DURSI (2000-). Joan Bladé, director técnico de Urquima. Ramon Capellades, director del LGAI (1999-). Gabriel Ferraté, rector de la Universidad Politécnica de Cataluña (1972-1976 y 1978-1994), director general de Universidades e Investigación y de Política Científica en el Ministerio de Educación y Ciencia (1976-1978), vicepresidente de la CIRIT (1980-1988) y actualmente rector de la Universitat Oberta de Catalunya (1995-). Rafael Foguet, consejero general del Grup Ferrer. Josep Font, vicerrector de la UAB (1976-1980, 1988-1993) y director general de Universidades (1993-1996). Josep Laporte, rector de la UAB (1976-1980), consejero de Educación (1988-1992) y comisionado de Universidades e Investigación (1992-1995). Narcís Majó, secretario de la CIRIT (1980-1988). Abel Mariné, director general de Universidades (1986-1990), secretario del Consejo Interuniversitario de Cataluña (1990-1991), vicepresidente de la CIRIT (1991-1993), director general de Investigación (en funciones) del comisionado para Universidades e Investigación (1993) y miembro de la Comisión de Asesoramiento y Seguimiento del Plan de Investigación de Cataluña-CIRIT (1993-1996). Andreu Mas Colell, comisionado de Universidades e Investigación (1999) y consejero del DURSI (1999-). Josep Maria Montangud, responsable de Coordinación Industrial del Departamento de Industria. Josep Oliver, vicerrector de Investigación de la UAB (1991-1993). Ramon Pascual, vicerrector de la UAB (1979), rector de la UAB (1980, 1986-1990), director general de Universidades (1980-1983), director del IFAE. Lluis Rovira, jefe del Gabinete Técnico de Investigación de la CIRIT (19922001). Carles Sans, director del CIDEM (1997-). Francesc Santacana, director de la Fundación Bosch i Gimpera (1986-). Josep Tarragó, director general del IRTA (1985-). Eugeni Terrer, gerente de Innovación Tecnológica del CIDEM (1997-). 
realizado atendiendo al estado y la orientación de los siguientes indicadores: en primer lugar, el volumen del presupuesto del gobierno regional destinado a la investigación académica o a la de enfoque más empresarial; el volumen total podría verse como una demostración de las preferencias generales o de la relevancia de esta política en la agenda de la Generalitat. En segundo lugar, el análisis de las acciones (planes, programas y demás), atendiendo a su naturaleza y destinatarios. Y, en tercer lugar, la política de creación de centros propios e infraestructuras, atendiendo fundamentalmente a la vinculación de los mismos y la naturaleza de su actividad ${ }^{4}$.

El argumento que puede explicar tanto el surgimiento como la varianza de las políticas, a partir de la comprensión del proceso político, se resume en: ideas, instituciones e intereses (Hall, 1989). En otro lugar (Sanz Menéndez, 1997) hemos aplicado este modelo a la explicación de la construcción institucional de la política científica y tecnológica española.

De forma general, y para este análisis que nos ocupa, se han considerado como factores explicativos, o variables independientes, los siguientes: las preferencias políticas del gobierno regional, las ideas y los modelos existentes sobre la política de $\mathrm{I}+\mathrm{D}$ de los cuales son portadores los diversos actores, los intereses en torno a esta política, con frecuencia organizados como policy community y los diseños institucionales 5 . También se ha considerado qué tipo de potencial científico-tecnológico se concentra en la región.

Podríamos establecer la hipótesis inicial de que los gobiernos tienen preferencias sobre qué políticas llevar a cabo, y que la explicación de la orientación de éstas últimas habría que buscarla en las primeras. Una hipótesis alternativa sería que los actores con intereses en estas políticas se movilizan para desarrollar sus propios modelos de interpretación, una vez que han conseguido controlar las instituciones responsables de las mismas.

\section{La emergencia y evolución de la política catalana de ciencia y tecnología}

Para entender el surgimiento de la política catalana de ciencia y tecnología debemos atender, en primer lugar, a factores estructurales relativos a los recursos previos que ya existían en la región, luego observar los procesos de difusión o imitación surgidos tanto de las transferencias como de las experiencias

4. Adicionalmente se han considerado el discurso político y la organización institucional, diseñada para separar o integrar a las distintas consejerías con intereses en la política global de $\mathrm{I}+\mathrm{D}$, así como para canalizar el acceso de los diferentes grupos (investigadores, organizaciones de investigación y empresas) a esta política.

5. Los diseños institucionales pueden ser tanto parte de la variable dependiente, debido a que, en este caso, la política regional de I+D se crea ex novo, como uno de los factores explicativos de esta política a lo largo del tiempo. Sin duda, los efectos de las diversas variables consideradas son distintos dependiendo de cuándo en el tiempo. Por ejemplo, si el sistema está poco maduro, el proceso determinante puede ser la formación de las preferencias políticas. Una vez construidas las instituciones políticas, en las cuales se integran los actores, la situación es otra. 
previas de los gestores y, finalmente, a las preferencias políticas manifestadas por los gobiernos autonómicos.

Aunque a mediados de los años 80, la mayor parte de la actividad y de los recursos en I+D se concentraban en Madrid, Cataluña representaba un 16,9\% del total de los gastos (Martín, Moreno y Rodríguez Romero, 1990). Un rasgo característico del sistema catalán de ciencia y tecnología es su equilibrio relativo entre los diversos actores de la $\mathrm{I}+\mathrm{D}$, con una mayor implicación del sector empresarial que en otras regiones españolas. En esta región el sector empresarial contribuía, en 1986, al gasto total en $\mathrm{I}+\mathrm{D}$ con un 78,6\%. Vista a través de sus resultados científico-técnicos, era la región con mayor peso en la producción de patentes (Sanz Menéndez y Arias, 1998) y la segunda por la producción científica (Maltrás y Quintanilla, 1992).

La concentración de recursos de investigación en la región sentaba las bases para el desarrollo de una política de intereses. En 1980, en Cataluña, estaban localizados una parte significativa de los recursos de investigación del país, aunque en el sector público la dependencia política de los actores se encontraba aún en la Administración central. Entonces existían tres universidades públicas, aunque con ramificaciones en todo el territorio regional. Hoy existen ocho universidades que componen el entorno universitario catalán (GC, 2000). Entre los actores públicos de I+D estaban los centros dependientes del CSIC en Cataluña, que hoy ascienden a diecisiete. Aunque el sector público de dependencia directa del Gobierno catalán a principios de los ochenta era inexistente, el mapa de los actores públicos de $\mathrm{I}+\mathrm{D}$ en Cataluña se ve completado por los centros de I+D que ha creado o potenciado la Generalitat, bien en colaboración con las universidades (por ejemplo el Centro de Supercomputación de Cataluña) o bien en el marco de los departamentos de la Generalitat (como por ejemplo, el Instituto de Investigación y Tecnología Agroalimentaria (IRTA), dependiente del Departamento de Agricultura, Ganadería y Pesca, o el Laboratorio General de Ensayos e Investigaciones (LGAI), dependiente del Departamento de Industria).

El surgimiento de la política catalana de investigación responde también a una opción política que busca la construcción de un marco competencial propio, en el que se incluyen las actividades de investigación y desarrollo. La primera experiencia directa de la nueva administración autonómica con las instituciones y las políticas de $\mathrm{I}+\mathrm{D}$ llegó de la mano del proceso de transferencias; la Generalitat obtuvo la titularidad de los centros de I+D del sector agrario, centros y recursos que un poco más tarde se constituyeron en el IRTA. Además, aunque no existía intención de imitar el modelo estatal con la creación de la CIRIT (Commisió Interdepartamental de Recerca i Innovació Tecnològica), quizá la experiencia en Madrid, en la política universitaria y de investigación, de alguno de los actores clave pudo ser determinante ${ }^{6}$ e influenciar la adopción de un modelo organizativo parecido a la CAICYT, tal y como

6. Ver nota 3. 
estaba vigente en 1980 (Sanz Menéndez, 1997). En ese modelo la preocupación esencial era la coordinación de las actuaciones y la resolución simultánea de los problemas, siempre con recursos escasos. La solución interdepartamental estaba diseñada.

Por último, las preferencias del gobierno de CiU de los primeros años eran claramente las de conformar una política de I+D coordinada entre los diferentes departamentos y con una cierta orientación empresarial o industrial. Sin embargo, en los ocho años en los que la Generalitat esperó la transferencia de los recursos estatales en política de $\mathrm{I}+\mathrm{D}$, se produjo un cambio sustancial derivado del traspaso a la Generalitat de la gestión de las universidades catalanas, con lo que el modelo de política que se proponía fue leído por los intereses académicos en clave de "financiación» de sus actividades de investigación.

A partir de 1988, las universidades ganaron la batalla institucional por el control de esta política y las preferencias del gobierno por una política de orientación industrial organizada interdepartamentalmente no pudieron llevarse a cabo. El resultado institucional fue la consolidación de la separación entre las esferas científica y tecnológica.

Las universidades catalanas eran unos actores muy influyentes en la política de $\mathrm{I}+\mathrm{D}$ catalana y la presión de los intereses académicos tuvo como resultado que, desde 1988, la CIRIT fuera un instrumento casi exclusivo de la Consejería de Educación, así como que el contenido de la política realizada desde esta institución se dirigiese a la satisfacción de los intereses académicos. Esto, a su vez, tuvo como consecuencia que las otras consejerías en las que se realizaba también una política de $\mathrm{I}+\mathrm{D}$ recelaran de «ponerse en manos» de la Consejería de Educación, y desarrollaran su propia política tecnológica por medio de la creación de centros propios y de una estrategia de apoyo a las empresas, sin apenas tener relación con la CIRIT.

En resumen, a lo largo del tiempo se produjo un desencuentro entre la preferencia original de los gobiernos de CiU hacia una política orientada a la industria y las fuertes presiones de los intereses académicos, en particular las universidades, concretada en un notable control institucional, para que se realizara una política más acorde con sus necesidades y estrategias. En definitiva, parece que se encontró una solución de compromiso entre la retórica "pro empresarial» del discurso político y unos esfuerzos reales concentrados en la realidad académica, aunque en los últimos años se ha ido consolidando un discurso construido sobre la "calidad» de la investigación, alternativo o complementario al discurso político de la competitividad.

Desde el punto de vista de la "prioridad política» otorgada a la investigación, y medida en términos de los esfuerzos presupuestarios, pueden observarse claramente dos momentos de gran crecimiento de los recursos, que se siguen de fases de relativo estancamiento e incluso retroceso (ver tabla 1 y gráfico 1 ). Además, el porcentaje del presupuesto total de la Generalitat de Cataluña dedicado a I+D ascendió notablemente, del $0,09 \%$ del presupuesto total de la Generalitat en 1988 al 0,46\% en 1996, en el contexto de cambios institucionales y organizativos de la Administración catalana. 
Tabla 1. Presupuestos de diversos organismos de fomento de la $\mathrm{I}+\mathrm{D}$ de la Generalitat de Cataluña (1981-1999) (en pesetas corrientes).

\begin{tabular}{|c|c|c|c|c|c|c|}
\hline Año & CIRIT & $\mathrm{DGU}+\mathrm{GTR}$ & DGU+SDGR & DGR & DGR/CIRIT & Total \\
\hline 1980 & 50 & & & & & 50 \\
\hline 1981 & 86,28 & & & & & 86,28 \\
\hline 1982 & 249,73 & & & & & 249,73 \\
\hline 1983 & 529,13 & & & & & 529,13 \\
\hline 1984 & 378,87 & & & & & 378,87 \\
\hline 1985 & 501,64 & & & & & 501,64 \\
\hline 1986 & 522,58 & & & & & 522,58 \\
\hline 1987 & 462,89 & & & & & 462,89 \\
\hline 1988 & 466,03 & & & & & 466,03 \\
\hline 1989 & 636,00 & & & & & 636,00 \\
\hline 1990 & 807,21 & $1.038,00$ & & & & 1845,21 \\
\hline 1991 & $1.447,81$ & $2.013,02$ & & & & $3.460,83$ \\
\hline 1992 & $3.100,90$ & & $2.429,64$ & & & $5.530,54$ \\
\hline 1993 & $3.520,90$ & & & $2.267,31$ & & $5.788,21$ \\
\hline 1994 & $3.576,68$ & & & $2.368,69$ & & $5.945,37$ \\
\hline 1995 & $3.708,16$ & & & $2.487,20$ & & $6.195,36$ \\
\hline 1996 & $3.274,18$ & & & $2.446,24$ & & $5.720,42$ \\
\hline 1997 & $3.329,18$ & & & $2.431,23$ & & $5.760,41$ \\
\hline 1998 & $3.319,41$ & & & $2.431,41$ & & $5.750,82$ \\
\hline 1999 & & & & & $5.803,92$ & $5.803,92$ \\
\hline
\end{tabular}

Fuente: Gabinete Técnico de Investigación (GTR) y Comisionado para las Universidades y la Investigación, 2000.

A continuación se analiza el surgimiento y la evolución de la política de investigación de la Administración catalana: una primera etapa de puesta en marcha y primeros pasos de la política en la que predomina una estructura interdepartamental de la I+D y en la que se configuran unas preferencias del gobierno regional tendentes hacia un modelo de orientación más industrial; una segunda etapa de reestructuración en la que se produce una separación institucional bastante pronunciada de la política científica y la tecnológica, y en la que se define una política de $\mathrm{I}+\mathrm{D}$ menos industrial de lo que el gobierno regional deseaba, debido a la presión de los intereses académicos; y una tercera etapa en la que claramente, y a raíz de la aprobación y desarrollo de los planes de investigación de Cataluña, se institucionaliza una política de orientación fundamentalmente académica y en la que hay una separación entre las áreas científica e industrial de la política, a pesar de la creación de instrumentos de articulación. 


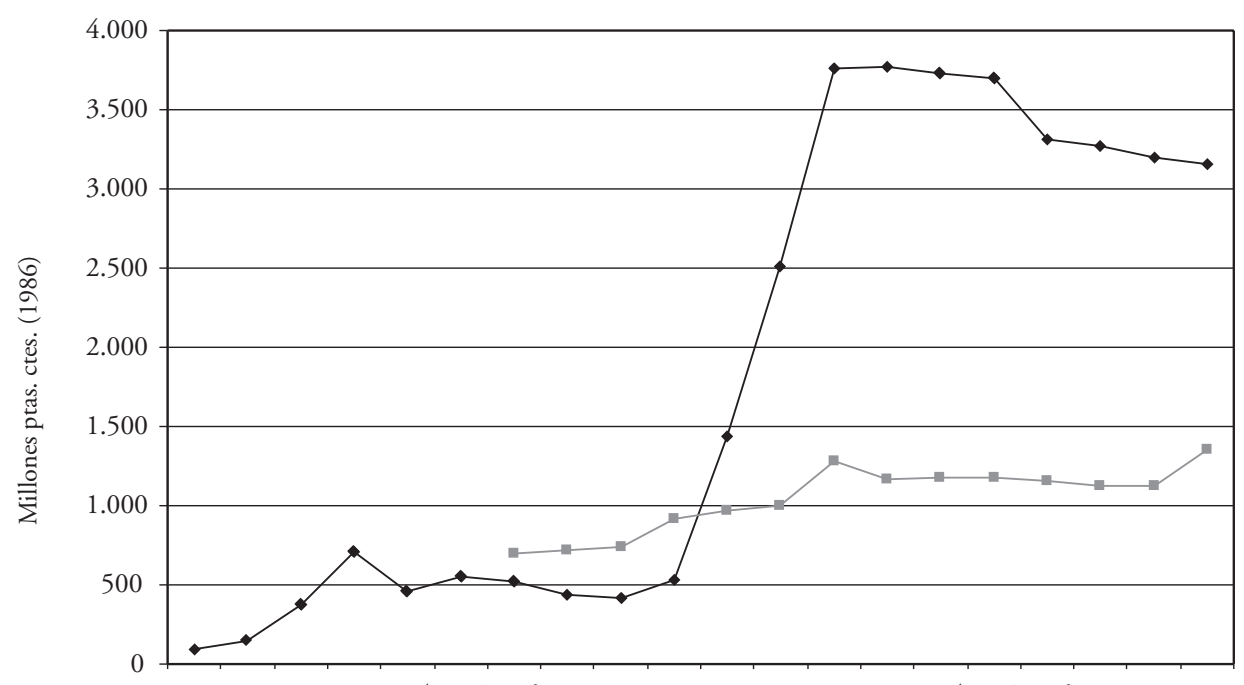

19801981198219831984198519861987198819891990199119921993199419951996199719981999

$\rightarrow-$ CIRIT + DGR $\rightarrow-$ IRTA

Gráfico 1. Evolución de los presupuestos de I+D de la Generalitat de Catalunya: CIRIT y DGR (investigación) e IRTA (investigación agraria). 1980-1999 (en pesetas corrientes 1986).

\subsection{Las preferencias iniciales del gobierno en politica de $I+D$ :}

Creación y primeros pasos de la CIRIT (1980-1988)

La primera etapa identificable en las actuaciones de la Generalitat en materia de $\mathrm{I}+\mathrm{D}$ se corresponde con las dos primeras legislaturas autonómicas. Esta etapa está caracterizada por la construcción de la política de investigación y sus instituciones. La decisión de construir instituciones interdepartamentales, y de desarrollar las primeras actuaciones de baja intensidad, mientras se esperaba y se batallaba por las transferencias desde el gobierno central, fueron eminentemente políticas.

Los gestores seleccionados para la construir la política procedían de la universidad $^{7}$, sin embargo preferían que la gestión de la política científica y tecnológica respondiese a un modelo coordinado y que sirviese también a la sociedad catalana. Pero la construcción de consejos asesores y la integración del «control político» de las universidades en la Consejería de Educación, comenzaron a crear las condiciones del cambio.

7. Ver nota 3 para el pasado de Gabriel Ferraté y Josep Laporte. Quizá la orientación proempresa de Ferraté estuviera relacionada con la singularidad de las relaciones que la UPC mantenía con el tejido industrial catalán y con su pasado en la Escuela de Ingenieros Industriales, de la que fue director entre 1969 y 1972. 
En esta primera etapa, que transcurre entre 1980 y 1988, dentro del gobierno catalán comenzaron a destacar tres actores esenciales en la definición de las estrategias: la CIRIT, la Consejería de Industria y la Consejería de Agricultura, beneficiada por las transferencias; pero el protagonismo se centró en la CIRIT, una estructura interdepartamental creada para la gestión de la política de I+D; fue entonces cuando se configuraron unas preferencias del gobierno regional tendentes hacia un modelo de orientación más empresarial-industrial.

La política de ciencia y tecnología catalana de la década de los ochenta estuvo protagonizada por el debate en torno a las transferencias del Estado a la Generalitat los recursos destinados a política científica y tecnológica ${ }^{8}$. A la espera de las transferencias, el exiguo presupuesto destinado por el gobierno regional para estos fines tuvo como resultado que se pusiera en práctica un modelo basado en actuaciones mínimas destinadas a la investigación académica, y con muy poca orientación industrial.

En las primeras elecciones autonómicas, en marzo de 1980, la coalición nacionalista moderada Convergència i Unió $(\mathrm{CiU})$ había obtenido la victoria, aunque no la mayoría absoluta; y pudo gobernar gracias al apoyo parlamentario de la Unión de Centro Democrático (UCD) y de Esquerra Republicana de Catalunya (ERC) ${ }^{9}$. Jordi Pujol, candidato de la coalición nacionalista, fue investido presidente de la Generalitat catalana.

El nuevo gobierno pretendía la presentación de un proyecto de ley de fomento y organización de la investigación en Cataluña, aunque consideraba que el momento de hacerlo dependería de la rapidez de las transferencias del Estado para este área. Desde la Generalitat se creía que el Estado, en particular en materia de ciencia y tecnología, iba a repetir los traspasos de la investigación agraria ya realizados, e iba a transferir las instituciones de investigación localizadas en Cataluña, así como los recursos correspondientes de los Presupuestos Generales del Estado a la Generalitat ${ }^{10}$.

En todo caso, la política científica y tecnológica no parecía una prioridad de la legislatura; desde el principio, y en una situación de espera de las transferencias, se creó la estructura de la CIRIT ${ }^{11}$. El origen de la idea de la CIRIT se encuentra en el seno de CiU. Tanto Gabriel Ferraté como Narcis Majó, futuros vicepresidente y secretario, respectivamente, de la CIRIT, creían que la política científica y tecnológica catalana debía estructurarse de manera interdepartamental. Consideraban que la investigación trascendía al ámbito universitario, al Consejo Superior de Investigaciones Científicas (CSIC) o al

8. Para una descripción y un análisis del marco legal de la investigación catalana, véase Puig Salellas (1991).

9. Véase Soler Llebaria (1997) para una panorámica de las elecciones autonómicas en Cataluña.

10. Discurso del candidato a la Presidencia de la Generalitat, Jordi Pujol. Diario de Sesiones del Parlamento Catalán, n. ${ }^{\circ}$ 4, 22 de abril de 1980.

11. Decreto 217/1980 de 5 de noviembre, de creación de la CIRIT (Comissió Interdepartamental de Recerca i Innovació Tecnológica) y el Consell Científic i Tecnològic (CCT), DOGC n. ${ }^{\circ}$ 93, 12 de noviembre de 1980. 
Instituto de Estudios Catalanes (IEC). Creían que el resto de consejerías de la Generalitat (agricultura, industria, etcétera) también realizaba y debía realizar investigación y, además y fundamentalmente, que la empresa catalana debía estar muy implicada en la investigación y el desarrollo. A pesar de que desde el principio hubo presiones muy fuertes por parte del Departamento de Educación, y de las propias universidades, para que la ciencia dependiera casi exclusivamente del mismo, estas reticencias se vencieron y, en noviembre de 1980, se creó la CIRIT dependiente de la Consejería de Presidencia. De esta forma, la comisión era interdepartamental, pues no dependía de ninguna consejería con intereses concretos y adoptaba una posición simétrica al emanar directamente de Presidencia. La CIRIT estaba presidida por el presidente de la Generalitat, pero, aparte del vicepresidente y del secretario, el resto de los miembros de la CIRIT eran los representantes de las diversas consejerías, además del presidente del Consejo Científico y Tecnológico (CCT). Para su puesta en marcha, la CIRIT contó con un presupuesto de tan sólo cincuenta millones de pesetas. Esta estructura era la que debía recibir las transferencias del Estado, pues se preveía que las transferencias no tardarían y con esta previsión no se le quería dotar de cuantiosos recursos propios de la Generalitat.

Para la CIRIT, la potenciación de la ciencia y la tecnología era una necesidad en Cataluña ${ }^{12}$. Para este fin se necesitaban tres elementos fundamentales: un marco legal adecuado, recursos para hacer una política determinada (personal, instalaciones, infraestructura y cooperación, así como un programa de actuación) y voluntad de acometer esa política. El marco legal estaba claro: la política científica debía tener una estructura interdepartamental equilibrada. El tipo de política que se pretendiera hacer, sin embargo, dependía del volumen de recursos existente. Con un presupuesto casi ridículo, apenas se podía hacer algo más que un programa mínimo compensador de la acción del Estado. A medida que el presupuesto fuera aumentando se afrontarían nuevas actuaciones. Esta estrategia de esperar las transferencias tuvo, a lo largo de estos ocho años, dos efectos relacionados: primero, que la política adoptada no tuviera una orientación industrial sino académica y, segundo, que los intereses académicos fuesen desarrollando ciertas expectativas, paralelas a lo que percibían en el ámbito nacional, que finalmente derivaron en una batalla por las instituciones que, en 1988, les dio el control sobre la gestión de la política, debilitándose, con ello, la estructura interdepartamental.

El panorama de la política de investigación catalana no fue muy distinto en la segunda legislatura, que comenzó tras las elecciones de abril de 1984, donde CiU volvió a vencer, esta vez por mayoría absoluta. Los traspasos en materia de investigación no habían llegado y no se sabía cuándo iban a llegar,

12. El 22 de octubre de 1982 el vicepresidente de la CIRIT, Gabriel Ferraté, compareció ante la Comisión de Política Cultural del Parlamento Catalán para explicar las líneas de actuación de la CIRIT (Diario de Sesiones del Parlamento Catalán, n. ${ }^{\circ} 44$ ). Este discurso es un buen reflejo de las ideas iniciales o los modelos de actuación en los que se basaba la CIRIT de los primeros años. 
aún así se tenía la confianza de que los iban a obtener. El discurso político ponía el acento en la orientación proempresarial. Consideraba que se había de seguir en la línea comenzada en la anterior legislatura y que además, desde el Departamento de Industria, había que dar incentivos y estímulos a la empresa privada para que innovara ${ }^{13}$. La CIRIT continuó durante esta segunda legislatura en la línea que había comenzado al principio de la década de los ochenta: con voluntad de una política de investigación de orientación empresarial, pero en la práctica, debido al exiguo presupuesto, haciendo una política de mínimos típicamente académica. Durante este periodo los recursos de la CIRIT se mantuvieron en torno a los quinientos millones de pesetas anuales e incluso, en algún momento, se contrajeron.

Si la situación de la CIRIT como organismo responsable de la política de investigación seguía estancada, en la Consejería de Educación se produjo un cambio importante con la transferencia de las universidades públicas a tutela de la Generalitat en 1986. Desde la universidad, las presiones seguían siendo constantes para que la CIRIT estuviera más relacionada con ellas y que no dependiera de Presidencia, sino de Educación ${ }^{14}$; el representante de Educación (normalmente el director general de Universidades) participaba en la CIRIT como un miembro más, pero este organismo continuaba con la misma política, de modo que el descontento, tanto en la Consejería de Educación como en las universidades, iba en aumento ${ }^{15}$.

Por otro lado, en la política de I+D del gobierno catalán también se producían cambios en otras dos instancias: en el área de la política hacia las empresas y en la tradicional política de investigación agraria. El Departamento de Industria, que no había tenido un papel muy importante en materia de investigación y desarrollo en la primera legislatura, empezó a desarrollar en la segunda legislatura cierta actividad. Así comenzó la denominada «primera fase hacia la innovación tecnológica» ${ }^{16}$, caracterizada por dar servicios básicos de certificación, ensayo y estandarización, aunque con escaso componente de innovación. La creación de centros, una de las dimensiones que hemos considerado relevantes para medir la orientación de las políticas, fue liderada por el Departamento de Industria. Así, se creaban centros e infraestructuras tecnológicas, como por ejemplo, en 1984, el Laboratori General d'Assaigs i Investigacions (LGAI), que pasó a ser una empresa dependiente de la Consejería de Industria, Comercio y Turismo ${ }^{17}$. La agencia regional de promoción, el CIDEM, también creado en 1985 por la

13. Discurso del candidato a la Presidencia de la Generalitat, Jordi Pujol. Diario de Sesiones del Parlamento Catalán, n.o 2, 29 de mayo de 1984.

14. Entrevistas a Gabriel Ferraté y Narcis Mayó.

15. Entrevistas a Ramon Pascual y Abel Mariné.

16. Entrevista con Carles Sans.

17. Ley de 28 de noviembre de 1984 de la Generalitat de Cataluña de creación del Laboratorio General de Ensayos e Investigación (LGAI). Hay que recordar que en esos primeros años de las transferencias en materia de industria y promoción hubo un fuerte activismo regional en la promoción del desarrollo regional desde los gobiernos autónomos, por ejemplo en Madrid (creación del IMADE), en Valencia (creación del IMPIVA), por lo que en este campo puede 
Consejería de Industria, iniciaba la primera fase hacia la innovación tecnológica ${ }^{18}$, y tenía por objetivo el progreso industrial, la mejora tecnológica y el aumento de la productividad y la competitividad catalana. A pesar de estos objetivos tan claros, no existía aún en el CIDEM un área de innovación, y sus actividades se concentraban en información, desarrollo, etc.; en realidad eran los años de «la incorporación de nuevas tecnologías en la empresa», más que los años de la política de innovación; dentro de las ayudas financieras a las empresas se incluía «la concesión de subvenciones para instalaciones industriales en Cataluña para la incorporación de tecnologías avanzadas» ${ }^{19}$.

El único área de investigación donde las transferencias fueron completas fue agricultura. En 1981 se recibieron las transferencias de los centros y las actividades del Instituto Nacional de Investigación Agraria (INIA) en Cataluña. Entre 1980 y 1985 la Consejería de Agricultura hizo un esfuerzo de ampliación y diversificación de la capacidad investigadora y en 1985 se creó por ley el Institut de Recerca i Tecnologia Agroalimentàries (IRTA), incluyendo los activos transferidos por el INIA, empresa de titularidad pública regida por derecho privado que se dedica a la investigación y la transferencia tecnológica en el sector agroalimentario ${ }^{20}$.

De hecho, mientras la Generalitat esperaba las transferencias en investigación, en 1986 las Cortes Generales aprobaron la Ley de Fomento y Coordinación General de la Investigación Científica y Técnica ${ }^{21}$. La aprobación de dicha ley supuso un varapalo para la Generalitat, debido a que el Estado, al amparo de la función de coordinación general, se constituía como órgano responsable de la planificación y ejecución de la política científica y tecnológica en todo el ámbito estatal. Esto quería decir que, sin perjuicio de la política de ciencia y tecnología que con fondos propios pudieran acometer las comunidades autónomas, el Estado no iba (salvo en contadas excepciones) a transferir sus recursos para este fin. La CIRIT, tal y como se había organizado, a la espera de las transferencias, quedó desprovista de sentido. La Generalitat y el Parlamento catalán plantearon un recurso de inconstitucionalidad por lo que consideraban una lectura estrecha y sesgada de la Constitución ${ }^{22}$.

identificarse un cierto proceso de aprendizaje colectivo e imitación en el que los «asesores tecnológicos» del CDTI jugaron un cierto papel de interés (véase Sanz Menéndez, 1997).

18. Se constituyó como una entidad de derecho público sujeta al derecho privado y adscrita a la Consejería de Industria y Energía.

19. Esta partida en 1985 supuso un gasto en ese año de 147,6 millones de pesetas (casi el 30\% de la cuenta de explotación del CIDEM), que deben ser considerados como gastos en equipamiento y equipos tecnológico más que como gasto en desarrollo de innovaciones tecnológicas (Memoria de actividades del CIDEM de 1985).

20. El IRTA comenzó en 1986 con un presupuesto de casi 700 millones de pesetas (repartido a partes iguales entre la explotación y la inversión) y, en 1999, este presupuesto alcanzaba los 2.496 millones de pesetas (de los que 1.823 millones corresponden a la explotación y 673, a las inversiones) (ver gráfico 1 ).

21. Para una descripción exhaustiva de las políticas del Estado, véase Sanz Menéndez (1997).

22. Este recurso fue desestimado en 1992. 
Lo cierto es que, a pesar de las preferencias políticas, la demora en dotar a la política catalana de I+D de recursos propios (el País Vasco, Andalucía o la Comunidad de Madrid asignaron cantidades propias con mayor rapidez), dificultó el desarrollo de policy communities compuestas por grupos distintos a los clientes académicos ${ }^{23}$, y así abrió la posibilidad de «apropiación» de la política de I+D - y de la propia CIRIT - por estos intereses. Sin duda, la coyuntura temporal es determinante para construir las capacidades de gobierno en una dirección u otra (Skocpol y Finegold, 1982). Cuando las universidades fueron transferidas, en 1986, los intereses académicos tenían experiencias y aprendían de los modelos del Estado que en ese momento estaban en ebullición. En Madrid, la CAICYT se reconvirtió en la CICYT y era un modelo de referencia para los investigadores catalanes que veían cómo la Secretaría General del Plan Nacional de I+D en Madrid era gestionada, junto con el nuevo Plan Nacional, por y para los intereses académicos (Sanz Menéndez, 1997). La imitación racional (Hedström, 1998) siguiendo las pautas aprendidas en sus relaciones previas con otras administraciones, fue una fuerza muy poderosa para defender sus intereses. Así, los investigadores catalanes fueron adquiriendo un cierto sentido de propiedad de la CIRIT. Ya sólo faltaba que la reorganización institucional hiciese de esas expectativas una realidad.

\subsection{La frustración de las preferencias del gobierno: la importancia}

de los intereses académicos en la búsqueda de nuevas instituciones para la política científica y tecnológica (1988-1992)

Entre 1988 y 1992, se produjo una reestructuración y reorganización del policy domain; se consolidó una separación institucional bastante pronunciada de la política científica y la tecnológica y además se definió una política de $\mathrm{I}+\mathrm{D}$ menos industrial de lo que el gobierno regional aparentemente deseaba, y ello principalmente como resultado de la presión de los intereses académicos.

En mayo de 1988, CiU volvió a lograr la mayoría absoluta y Pujol continuó siendo el presidente de la Generalitat. El discurso era el mismo, el gobierno insistía en apoyar las iniciativas de investigación y desarrollo y de transferencia de tecnología: «el objetivo (de política científica y tecnológica), más que aspirar al nivel más alto, que hoy día no está a nuestro alcance, es que la nueva tecnología se aplique a la actividad industrial y agraria catalana» ${ }^{24}$. Aunque

23. De hecho, tras la salida de Ferraté y Majó de la CIRIT, que aportaban la visión de los ingenieros de las relaciones con la sociedad y la economía catalanas, el grupo de responsables de las políticas de $\mathrm{I}+\mathrm{D}$ se hizo más coherente y estable. Era un grupo relativamente cerrado, prácticamente todos era catedráticos o investigadores del CSIC, y estuvieron muchos años en los cargos de responsabilidad, que incluso se permutaban. Ver nota 3.

24. Discurso del candidato a la Presidencia de la Generalitat, Jordi Pujol. Diario de Sesiones del Parlamento Catalán, n.o 2, 21 de junio de 1988. 
todavía se tenía la esperanza de lograr las transferencias del Estado para la política científica y tecnológica regional, se inició una importante reorganización institucional de la ciencia y la tecnología catalanas.

La influencia de los intereses académicos tuvo como efecto un cambio institucional de consecuencias notables. Las presiones de las universidades para que la CIRIT pasara a depender de la Consejería de Educación habían sido constantes en la anterior etapa y, en 1988, las presiones dieron su fruto ${ }^{25}$, ya que se aprobó un decreto mediante el que el presidente de la Generalitat dejaba de ser el presidente de la CIRIT, pasando este cargo al consejero de Educación ${ }^{26}$. En el terreno de la gestión, esto significó que Josep Laporte ${ }^{27}$, nuevo consejero de Educación, pasaba a ser presidente de la CIRIT, y tanto Gabriel Ferraté como Narcís Majó dejaban sus responsabilidades, de vicepresidente y secretario de la CIRIT respectivamente. Según los cargos salientes, esta transformación institucional mermó la capacidad interdepartamental de la CIRIT, pero se optó por un modelo en el que se evitara la falta de sintonía e incluso el enfrentamiento entre la CIRIT y la Dirección General de Universidades, que hasta el momento habían estado "descoordinadas», es decir, parecían dos agencias de política científica independientes y esta descoordinación se percibía como negativa ${ }^{28}$.

La Consejería de Educación había sido poco activa en materia de política científica entre 1980 y 1988. Administrativamente, esta función había correspondido a la CIRIT, que estaba desligada de Educación. La labor de la Dirección General de Universidades (DGU) se había centrado en los primeros años en el establecimiento de servicios científico-técnicos y en la incorporación de investigadores españoles en el extranjero. Además, esta Dirección actuaba fundamentalmente por medio de la concesión de becas predoctorales.

A partir de 1988, la Consejería de Educación, en la que se integró la CIRIT, tuvo como objetivo la potenciación de la investigación en las universidades $^{29}$. Se quería aumentar el nivel de la investigación universitaria en Cataluña. Al contar con pocos recursos, y empezar a tener expectativas de que los recursos del Estado central no iban a llegar, se optó por no hacer una "política de financiación de proyectos». Se llevaron a cabo acciones de tipo «intersticial», es decir, acciones encaminadas a ayudar a los grupos de investigación para que estuvieran en las mejores condiciones posible para competir por los recursos del Plan Nacional, e incluso en los programas europeos. Se pensaba que la CICYT debía ser la encargada de financiar proyectos de I+D y que la misión del gobierno regional consistía en tratar que sus grupos de investigación fueran lo más competitivos posible. Se continuó e incrementó

25. Entrevistas a Gabriel Ferraté y Narcis Majó.

26. Decreto 144/1988, de 5 de julio.

27. Era también académico y médico, como Pujol.

28. Entrevista a Artur Bladé.

29. Entrevista a Josep Laporte. 
la política de becarios de investigación, la de estancias en el extranjero y las ayudas para infraestructuras.

Un cambio muy significativo se produjo en este periodo fruto del incremento de los presupuestos para I+D, que, entre 1990 y 1992, fue espectacular. La voluntad política de dar prioridad presupuestaria a la ciencia y la tecnología se produjo cuando el control institucional de esta política estaba ya en la esfera de Educación ${ }^{30}$.

En este periodo las otras dos consejerías activas en estas áreas continuaron su andadura más independiente, si cabe, dada la reorientación de la CIRIT hacia Educación. Durante este periodo, de 1988 a 1992, la Consejería de Industria continuó con "la primera fase hacia la innovación tecnológica», es decir, con la estrategia basada en la provisión de servicios muy básicos de certificación, ensayo y estandarización; además del LGAI, se crearon otros centros como, en 1990, el IDIADA (Institut d'Investigació Aplicada de l'Automóbil) con la misión de apoyar a sus clientes, las empresas, en las actividades relacionadas con el desarrollo de productos a través de la provisión de los servicios de ingeniería, ensayos y homologación. El CIDEM, en esta época, se enmarcaba dentro de esta línea de actuación general de la Consejería de Industria: concedía una importancia vital a los centros de transferencia de tecnología. Al contrario que en años anteriores, en este periodo tenía un área de tecnología, lo que puede significar una mayor concienciación sobre este tipo de cuestiones ${ }^{31}$.

Fruto del cambio institucional —el paso de la CIRIT a la Consejería de Educación-, en Cataluña se comenzó a configurar un sistema con una progresiva separación de la política científica, por un lado, y la política tecnológica, por otro, todo ello aparentemente en contra de las preferencias expresadas en el discurso de CiU. La política científica se encargaría de la ciencia más básica, fundamentalmente realizada en universidades y centros de investigación, y sería diseñada y gestionada desde la Consejería de Educación, de la que dependía la CIRIT, con gran influencia de la Dirección General de Universidades. Asimismo, la política tecnológica, la investigación más aplicada, sería competencia de la Consejería de Industria y, muy especialmente, del CIDEM, así como de otras consejerías sectoriales con competencias, tales como la de Agricultura a través del IRTA, que ejecutaba el servicio de investigación para un sector muy fragmentado.

30. Si en 1988 la CIRIT contaba con 466,0 millones de pesetas, en 1992 su presupuesto había ascendido a 3.100,9 millones de pesetas (en 1990 era de 807,2 millones). También aumentó el presupuesto de investigación de la Dirección General de Investigación, donde el mayor incremento corresponde a ese periodo (en 1990 se dedicaban 1.038 millones de pesetas y, en 1992, 2.429,6 millones de pesetas) (tabla 1).

31. Memoria de actividades del CIDEM de 1990. 


\subsection{La institucionalización de la reorientación: la consolidación de la separación de la política de ciencia y tecnología y los planes de investigación de Cataluña}

Con la CIRIT en la órbita de la Consejería de Educación, el discurso político comenzó a hacerse más consistente con la realidad de las actuaciones. Durante la tercera etapa, que transcurre entre los años 1992 y 2000, y a raíz de la aprobación y el desarrollo de los planes de investigación de Cataluña, se configuró una política de $\mathrm{I}+\mathrm{D}$ dominada por la orientación académica y en la que hay además una clara separación entre estas dos áreas de la política, la científica y la tecnológico-industrial; a pesar de que en el discurso político seguía dominando la preferencia por una política más articulada.

En las elecciones de 1992, CiU conquistó de nuevo la mayoría absoluta y Jordi Pujol volvió a ser presidente de la Generalitat de Cataluña. El gobierno en su conjunto tenía como objetivo general hacer de Cataluña una región con una industria más competitiva, para lo que debía hacerse un esfuerzo en $\mathrm{I}+\mathrm{D}$ y en la transferencia de tecnología desde las universidades y los centros de investigación a las empresas. Desde el punto de vista organizativo, se propuso la creación de un comisionado encargado de las universidades y la investigación que contribuiría a estrechar la relación entre educación e investigación ${ }^{32}$.

Así, en diciembre de 1992, se aprobó un decreto mediante el que se creaba el Comisionado de Universidades e Investigación ${ }^{33}$. Este órgano surgió de la división de la Consejería de Educación, dejando la educación no universitaria en la antigua consejería, y pasando la educación universitaria y la investigación al Comisionado ${ }^{34}$. Desde el punto de vista de la investigación, el Comisionado tenía una Dirección General de Investigación (DGR) y la CIRIT era presidida por el comisionado.

Josep Laporte, Consejero de Educación anterior, pasó a ocupar el Comisionado al escindirse y expresó los objetivos ante el Parlamento catalán cuando explicaba el Plan de Investigación de Cataluña ${ }^{35}$. La construcción del nuevo discurso estaba en marcha; la investigación no sólo era muy importante desde el punto de vista económico, como factor de competitividad, sino desde el punto de vista social. Se pretendía establecer las bases para mejorar la calidad y la competitividad del sistema de ciencia y tecnología catalán a través del Plan de Investigación de Cataluña. Este plan tenía como objetivo potenciar la investigación de base en universidades y en centros de investigación,

32. Diario de Sesiones del Parlamento Catalán, n.o 2, 8 de abril de 1992.

33. Decreto 318/92 de 28 de diciembre.

34. Este Comisionado dependía de Presidencia de la Generalitat, y, desde el punto de vista funcional, era similar a una consejería, pues, por ejemplo, el comisionado podía asistir a las reuniones del gobierno cuando fuera especialmente convocado. Este tipo de arreglo institucional se adoptó, en 1997, en Galicia con la creación de la Secretaría General de I+D (Fernández, Sanz Menéndez y Cruz, 2003).

35. Diario de Sesiones del Parlamento Catalán, n. ${ }^{\circ}$ 112, de 14 de octubre de 1993. 
delimitar unas líneas prioritarias de actuación, así como estimular a las empresas para que gastaran más en $\mathrm{I}+\mathrm{D}^{36}$.

La política científica y tecnológica catalana se consolidó con el Pla de Recerca de Catalunya (1993-1996) (GC, 1993), que tenía como objetivo general asegurar un desarrollo equilibrado y eficaz del sistema de $\mathrm{I}+\mathrm{D}$ catalán, erigiéndose como el instrumento de coordinación de la política de la Generalitat en materia de investigación científica y desarrollo tecnológico. El Plan tenía su ámbito principal de aplicación en el sector público, aunque además pretendía estimular las actuaciones complementarias que potenciaran sectores productivos de interés, y que incrementaran y optimizaran los recursos ajenos. Este plan presentaba una cierta orientación académica, pues estaba muy enfocado hacia las universidades y centros de investigación y su principal objetivo, tal y como se desprende del análisis de sus programas, era el incremento de la calidad investigadora en estas instituciones.

El Plan de Investigación no pretendía en absoluto copiar el modelo de los planes nacionales o europeos, tal y como se había hecho en otras comunidades autónomas; de hecho era un plan complementario y se descartó desde el principio realizar convocatorias de proyectos, tal y como se hacía en el Plan Nacional de I+D o en el Programa Marco de I+D; es decir, se renunciaba a conceder financiación por proyectos. Esto evitaba duplicidades en la financiación, y fomentaba la competitividad por los recursos, tanto nacionales como extranjeros; es decir, se trataba de invertir en personal de apoyo, infraestructura, becarios, etc., para que los equipos de investigación captaran los máximos recursos externos ${ }^{37}$.

A principios de 1994 se crearon la Comisión de Asesoramiento y Seguimiento y el Consejo de Evaluación Científica y Técnica como órganos de apoyo a la CIRIT para el desarrollo del Plan. Estos órganos estaban formados por personalidades significativas del mundo de la investigación y de los sectores social, cultural y económico. Sin embargo, a pesar del reconocimiento formal en este tipo de órganos de la política de ciencia y tecnología catalana del mundo industrial y empresarial, lo cierto es el escaso papel que estos sectores han desempeñado en comparación con las universidades (en especial, los vicerrectores de investigación $)^{38}$.

El Plan se estructuraba en dos grandes programas: el de Fomento General de la Investigación y el de Líneas Prioritarias. El primero de estos programas se refería a la investigación básica y aplicada de calidad y estaba constituido por acciones de carácter horizontal, poniendo el énfasis en conseguir una cobertura adecuada de las diferentes áreas del conocimiento e identificando tanto líneas de trabajo como grupos y centros de calidad, con el fin de promocionar sus actividades en el contexto más amplio de la investigación internacional. El programa de líneas prioritarias tenía como meta la potenciación de

36. Entrevista a Josep Laporte.

37. Entrevista a Joan Albaigès.

38. La mayor parte de los entrevistados coincide en señalarlo. 
determinadas líneas que quedaran desatendidas o que conviniera reforzar de acuerdo con el potencial, las necesidades y las características del entorno catalán. Las evidencias de retraso tecnológico en el tejido industrial catalán hacían aconsejable que el Plan de Investigación dedicara una especial atención a todo lo que contribuyera a impulsar más decididamente su competitividad. Por último, el Plan de Investigación creaba la Red de Centros de Referencia de la Generalitat, con el fin de reforzar el potencial científico y tecnológico del país, de forma que pudiera actuar como verdadero motor de la competitividad en los distintos subsectores económicos. Se trataba, con esta medida, de reunir a grupos de investigación de tamaño adecuado y de alta calidad, en torno a unos objetivos concretos, definidos en un contrato programa financiado por el Plan.

El programa de fomento general de la investigación tuvo como principales ámbitos de actuación la formación de recursos humanos y el apoyo y el fomento de la investigación. La política de becas y ayudas de la Generalitat de Cataluña en 1993 y 1994 reflejaba las líneas principales del plan, tendentes, como se ha comentado con anterioridad, hacia una política basada en un modelo de naturaleza académica. Los recursos dedicados a estas partidas (becas predoctorales en España y en el extranjero, infraestructura científica, etcétera) fueron muy cuantiosos $^{39}$. El programa de líneas prioritarias, y en concreto en la línea prioritaria de desarrollo tecnológico, no tuvo ni mucho menos el respaldo presupuestario del programa de fomento general de la investigación ${ }^{40}$.

En el periodo de 1992 a 1995 se puede observar un crecimiento moderado, incluso un estancamiento real del presupuesto de la CIRIT, que, en 1992, era de 3.100,9 millones de pesetas y alcanzó los 3.708,2 millones en 1995. Lo mismo ocurre con el presupuesto de la Dirección General de Investigación, dado que éste disminuyó entre estos dos años, de 2.429,6 millones de pesetas a 2.267,3 millones de pesetas. Excepto entre estos dos años, el presupuesto de la Dirección General de Investigación aumentó levemente hasta 1995. En este último año ascendió a 2.487,2 millones de pesetas; en comparación con estas

39. Entre 1993 y 1996 se concedieron 3.610 becas para la formación de personal investigador (y técnico) en Cataluña, en su mayoría becas predoctorales, con un gasto de 7.112 millones de pesetas. Las becas de formación de personal en el extranjero concedidas ascendieron en ese periodo de tiempo a 1.060 (unas $300 \mathrm{al}$ año), con un gasto asociado de 1.493 millones de pesetas. El Plan de Investigación asignó 3.233 millones de pesetas para la mejora de la infraestructura científica y su mantenimiento de las universidades y centros públicos de investigación. Entre 1993 y 1996 se concedieron 2.272,1 millones de pesetas para la contratación de técnicos de apoyo o de doctores, así como para la adquisición de equipamiento menor o material fungible de 427 grupos de investigación catalanes (269 grupos consolidados y 158 grupos financiados).

40. Las ayudas a las empresas para la incorporación contractual de titulados universitarios a los departamentos de $\mathrm{I}+\mathrm{D}$ y las ayudas al desarrollo de proyectos de innovación tecnológica recibieron 583 millones de pesetas (de la CIRIT) en 1993 y 1994. En 1995 se acordó que estas ayudas fueran conjuntas entre la CIRIT y el CIDEM (y que las gestionara el CIDEM). Por ello, en ese año el presupuesto conjunto dedicado a esta partida fue de 460 millones de pesetas. 
cifras, el CIDEM gastó poco más del 15\% de esas cantidades en subvenciones a las empresas para la innovación.

En la Consejería de Industria, por medio del CIDEM, se produjo en 1995 un cambio significativo en el planteamiento de la política tecnológica y se pasó de poner énfasis en la provisión de servicios muy básicos de certificación, ensayo y certificación, a "la segunda fase hacia la innovación tecnológica» ${ }^{41}$. Esta fase implicaba incentivar a las empresas para aumentar la innovación tecnológica. No se trataba ya de crear centros para ayudar a las empresas a través de determinados servicios, sino de dar incentivos a las propias empresas para que innovaran. En un primer momento esto se hizo a través de subvenciones a las empresas para proyectos desarrollados por las mismas.

En todo caso, en 1995, por primera vez se lanzaba una convocatoria de ayudas conjunta CIRIT-CIDEM ${ }^{42}$ para el fomento de la innovación tecnológica en las empresas, consistente en ayudas a las empresas para la investigación industrial, aplicada y desarrollo tecnológico, así como ayudas para incorporación contractual de titulados universitarios a los departamentos de I+D de las empresas situadas en Cataluña ${ }^{43}$. Los destinatarios de estas ayudas eran PYMES catalanas que desarrollaran proyectos de investigación industrial para crear nuevos productos y procesos y así aumentar su competitividad. Como se ha dicho, la cuantía, aunque no era despreciable, no era equivalente a lo gastado en el Plan de Investigación en promoción del conocimiento ${ }^{44}$.

Las elecciones de noviembre de 1995 supusieron la pérdida de la mayoría absoluta para CiU. El resultado de estas elecciones forzó a este partido a acuerdos puntuales con otros partidos, fundamentalmente ERC y el PP (aunque puntualmente también fue apoyado por el PSC-PSOE). El gobierno iba a prestar más atención, entre otras cuestiones, a la investigación. En particular, se destacaba la importancia de las empresas, en colaboración con las universidades, en el desarrollo de I+D e innovación ${ }^{45}$.

En 1995, Joan Albaigès, que hasta ese momento era el director general de Investigación, pasó a estar al frente del Comisionado de Universidades e Investigación, en sustitución de Josep Laporte. El periodo que transcurrió de

41. Entrevista con Carles Sans.

42. El 24 de abril de 1995 se publicó la resolución sobre la apertura de la convocatoria de las ayudas CIRIT-CIDEM para la realización de proyectos de I+D. Además, el 19 de julio de 1995 se publicó la resolución sobre la apertura de la convocatoria para concesión de ayudas CIRIT-CIDEM para la realización de proyectos de I+D a empresas que incorporaran titulados superiores de universidad para llevarlos a cabo, con el objetivo de favorecer la investigación industrial y proporcionar experiencia a los jóvenes titulados.

43. Además, entre 1993 y 1996 se concedieron 169 ayudas a la incorporación de titulados, con un presupuesto asociado de 175,9 millones de pesetas, y 320 ayudas al desarrollo de proyectos, con un presupuesto de 921,3 millones de pesetas.

44. A lo largo del año 1995 se otorgaron 50 ayudas por importe de 152 millones de pesetas sobre una inversión subvencionable de 965 millones, que movilizaba una inversión total de 1.200 millones de pesetas.

45. Discurso del candidato a la Presidencia de la Generalitat, Jordi Pujol. Diario de Sesiones del Parlamento Catalán, n. ${ }^{\circ}$ 2, 13 de diciembre de 1995. 
1995 a 1999 no conllevó ningún cambio sustancial en la política desarrollada por el Comisionado, que estuvo marcada por la continuidad. En 1997 se presentó el segundo Plan de Investigación (1997-2000), continuista respecto al primero, aunque en él se intentó, por un lado, llegar a un nivel de detalle mayor en las actuaciones y, por otro, atender más a la demanda de los sectores productivos, aunque el énfasis en el desarrollo tecnológico era más bien retórico.

Los objetivos generales del II Pla de Recerca de Catalunya (1997-2000) (GC, 1997) no eran muy distintos de los planteados en el I Plan: los programas de fomento general de la investigación y de desarrollo y transferencia de tecnología, constituían herramientas para el desarrollo de los objetivos en las distintas áreas. El primer programa estaba claramente orientado al ámbito universitario (becas de formación, ayudas de apoyo a la investigación, etcétera) y el segundo, más orientado a la industria (ayudas a las empresas para recursos humanos de $\mathrm{I}+\mathrm{D}$, acciones movilizadoras de desarrollo y transferencia de tecnología, etcétera). La política de ayudas de la Generalitat de Cataluña de 1997 a 1999 reflejó los objetivos del nuevo plan, que, como se ha comentado con anterioridad, eran de naturaleza principalmente académica, aunque, quizá, en menor medida que en el primer Plan de Investigación.

Por otro lado, el periodo de 1995 a 1998 fue un periodo de ligero descenso y posterior estancamiento del presupuesto de la CIRIT. Si el presupuesto de este organismo fue de 3.708,2 millones de pesetas en 1995, en 1996 bajó a 3.274,2 millones, llegando en 1998 a la cifra de 3.319,4 millones. Esta reducción entre 1995 y 1996 correspondió exclusivamente a la CIRIT, y no al presupuesto de la Dirección General de Investigación, que permaneció estable, en estos años, en torno a los 2.400 millones de pesetas.

Desde la Consejería de Industria, en particular desde el CIDEM, se continuó incentivando a las empresas para aumentar la innovación tecnológica, principalmente a través de subvenciones a proyectos. La misión era desarrollar un entorno que favoreciera la innovación tecnológica entre las empresas, teniendo como objetivos concretos el fomento de la cultura de la innovación entre las empresas, la colaboración para resolver sus retos tecnológicos, favorecer la transferencia de tecnología y el acceso a una oferta tecnológica adecuada, completar la oferta tecnológica con centros de innovación orientados a la demanda, y asesorar sobre los mecanismos institucionales de apoyo a la innovación. El programa estrella continuó siendo las ayudas CIRIT-CIDEM a empresas para la ejecución de proyectos de I+D, que supusieron un gasto, en 1999, de 300 millones de pesetas, con una inversión total movilizada de 1.500 millones.

La Generalitat estaba, por fin, desarrollando sus preferencias originales, pero coincidía con un momento de estancamiento presupuestario, que pasaba a reflejarse en el peso de la política de I+D en los presupuestos de la Generalitat catalana, que empezó a disminuir a partir de 1996. En ese año representó el 0,46\% del presupuesto de la Generalitat, mientras que en 1999 tan sólo se dedicó el 0,39\% del presupuesto general de la Generalitat.

La división entre política científica, por un lado, y política tecnológica, por otro, que se estableció con claridad en Cataluña a partir de 1988, es aún bas- 
tante pronunciada. En la actualidad se cuenta con dos planes independientes, aunque relacionados: el III Plan de Investigación de Cataluña. 2001-2004 (GC, 2001a) y el I Plan de Innovación de Cataluña. 2001-2004 (GC, 2001b). El primero es impulsado desde el DURSI ${ }^{46}$ y el segundo, desde la Consejería de Industria (CIDEM), lo que significa un reconocimiento de las dificultades de desarrollar dos tareas que exigen fondos y recursos, cuya suma es cero, desde la misma organización. Por otro lado, según Mas Colell, se ha optado desde el DURSI por dejar fuera a la investigación más ligada a la empresa, aunque estableciendo puentes con ella, porque se piensa que esta política más tecnológica debe realizarse desde la Consejería de Industria. El objetivo fundamental del DURSI, y por lo tanto de la política científica, es fomentar la calidad, la excelencia en la investigación. Para ello se desarrollan medidas entre las que la más novedosa es la construcción de centros y la creación de plantillas del personal investigador dependiente de la Generalitat.

Desde el punto de vista de la Consejería de Industria, y por lo tanto de la política tecnológica, el objetivo es incrementar la innovación de las empresas, aunque desviándose de las ayudas directas que predominaron en una etapa anterior; ahora se va a enfatizar la subcontratación de la investigación, principalmente a las universidades y los centros de investigación, como mecanismo para contribuir a la innovación en las empresas. En el fondo parece que la política científica de orientación académica domina en Cataluña e incluso la forma en que se construyen algunos instrumentos que se proponen para la política empresarial, otorgan a los centros públicos de $\mathrm{I}+\mathrm{D}$ y universidades un papel dominante.

\section{Conclusiones}

Este trabajo ha tenido como objetivo describir la emergencia y las opciones tomadas por el gobierno catalán a favor de determinadas políticas de $\mathrm{I}+\mathrm{D}$ e innovación. Las preguntas de investigación que han vertebrado la construcción del argumento son: ¿por qué, y en qué circunstancias, surgen las intervenciones de los gobiernos regionales en materia de $\mathrm{I}+\mathrm{D}$ y sobre todo qué forma adoptan? Por otro lado, nos interesaba saber, ¿`cuáles son los factores

46. En el año 2000 se produjo una transformación organizativa de la política de ciencia y tecnología muy relevante. El Comisionado de Universidades e Investigación se convirtió en una consejería independiente, la Consejería de Universidades, Investigación y Sociedad de la Información (DURSI). En este momento se vio que convertir el Comisonado en Consejería podía concederle a la investigación mayor importancia de cara a la opinión pública y, además, dotar al consejero de mayor fuerza de cara a los otros consejeros y de cara a determinados sectores, como los rectores. De esta Consejería pasaron a depender la Dirección General de Investigación y la CIRIT. La CIRIT pasó a ser, como en los orígenes, presidida por el presidente de la Generalitat, aunque la vicepresidencia recaía en el consejero del DURSI. Al frente de esta Consejería se colocó al reputado economista, académico nato, Andreu Mas Colell, quien había sido nombrado comisionado de Universidades e Investigación poco antes. 
más influyentes en la forma que la política adopta y, en definitiva, en las opciones que se toman?

Para responder a estas preguntas se ha utilizado un marco analítico que sitúa la comprensión del proceso político en el centro de la explicación y que combina las nociones de ideas, instituciones e intereses para dar cuenta del proceso por el que se eligen determinadas políticas de I+D frente a otras.

La Generalitat catalana fue una de las primeras administraciones regionales españolas en poner en marcha una política de ciencia y tecnología propia, distinta de la estatal, tal y como se establecía en su Estatuto de Autonomía de 1979. En el discurso político emergió, a principios de los ochenta, una cierta preferencia por una política de I+D más ligada al mundo empresarial. Desde el Gobierno de la Generalitat de Cataluña se pusieron en marcha iniciativas de política de $\mathrm{I}+\mathrm{D}$ dirigidas tanto al mundo académico como el empresarial.

La política de ciencia y tecnología desarrollada por el gobierno regional catalán desde principios de los años ochenta ha sido una política con contenidos en los que, a pesar de las preferencias manifestadas en el discurso político y concretadas en una primera fase en el diseño de instituciones interdepartamentales, sin embargo, ha predominado un modelo académico de política sobre el de orientación empresarial. Asimismo, en términos organizativos e institucionales, en la Administración autonómica la política científica ha estado, por norma general, separada y diferenciada de la política tecnológica.

Pero la principal razón de que la política de $\mathrm{I}+\mathrm{D}$ catalana no siguiera un modelo más industrial ligado al mundo empresarial, como era el deseo inicial del gobierno de $\mathrm{CiU}$, fue la presión que ejercieron las universidades catalanas para que, tanto el diseño institucional como el contenido de la política les favoreciera y se adaptara a sus necesidades. Además, los responsables de las políticas de $\mathrm{I}+\mathrm{D}$ tenían una trayectoria esencialmente académica, lo que contribuyó a una policy community dominada por los intereses académicos.

Las preferencias del gobierno de CiU de los primeros años eran claramente las de conformar una política de $\mathrm{I}+\mathrm{D}$ coordinada entre los diferentes departamentos y con una cierta orientación industrial. La creación de la CIRIT en 1980 dependiente de Presidencia respondía a estos objetivos. Sin embargo, la opción tomada de esperar las transferencias del Estado, suponía no asignar recursos de los presupuestos catalanes al fomento de la I+D, por lo que apenas se pudo realizar política alguna. Esa decisión gubernamental de no dotar de prioridad presupuestaria a sus preferencias, a la espera de las transferencias, creó un espacio para la elevación de expectativas en las universidades, expectativas intertemporales que fueron determinantes en la batalla de éstas últimas por el poder institucional a partir de 1988. Cuando a partir de entonces la asignación de recursos propios a $\mathrm{I}+\mathrm{D}$ adquirió prioridad presupuestaria, el control de la gestión estaba ya en el ámbito educativo, cuyos intereses ofrecieron soluciones e interpretaciones alternativas a las preferencias manifestadas. 
Así pues, debido a la fuerte presión de las universidades, en 1988 la CIRIT pasó a depender de la Consejería de Educación, lo que supuso una merma en su capacidad interdepartamental. La Dirección General de Universidades y la CIRIT conformaron una política científica a partir de entonces de corte claramente académico y fuertemente influida por los centros públicos de investigación, en especial por las universidades, y de hecho gestionada por personal salido de esos centros.

Cataluña, con relación al resto de regiones españolas, presenta y presentaba, en la década de los años ochenta, un peso importante en las actividades de $\mathrm{I}+\mathrm{D}$, tanto de empresas como de centros públicos de investigación y universidades. Sin embargo, a pesar de la importancia de las empresas en la I+D lo cierto es que, tal y como se ha observado en este caso, estos actores no se movilizaron ni presionaron a los gobiernos suficientemente. Las empresas, aunque con gran importancia en el sistema catalán de ciencia y tecnología, no contrarrestaron la presión ejercida por los intereses académicos debido a que las políticas de $\mathrm{I}+\mathrm{D}$ no constituyeron para ellas una prioridad y su dependencia de los recursos públicos era menor.

Finalmente, una peculiaridad de la política de I+D catalana, sobre todo en relación con el resto de regiones españolas, es el hecho de que la Generalitat nunca se planteara explícitamente la imitación del modelo estatal, en particular de los planes nacionales ${ }^{47}$, cuya pieza clave era la financiación de proyectos de I+D. En Cataluña se planteó un modelo diferente consistente en la creación de centros, el apoyo a las políticas de fomento de los recursos humanos y en ayudas para que los grupos de investigación estuvieran en las mejores condiciones para competir por los fondos tanto nacionales como europeos.

El balance de los recursos financieros utilizados por la Generalitat, a lo largo de las décadas de 1980 y 1990, para financiar las actividades académicas y las empresariales en el campo de la $\mathrm{I}+\mathrm{D}$ es significativamente distinto. Se podría estimar que más del $80 \%$ de los recursos regionales se dedicaron a la política de $\mathrm{I}+\mathrm{D}$ de orientación académica, sólo compensada por el gasto institucional de los centros propios de la Generalitat para facilitar servicios a las empresas.

La política catalana de I+D es un caso de gran interés analítico. En primer lugar, este caso ilustra cómo la creación de instituciones, sin venir acompañada del otorgamiento de prioridad presupuestaria, no garantiza la realización de las preferencias políticas. En segundo lugar, el caso catalán pone de manifiesto cómo el horizonte temporal de la toma de decisiones gubernamental tiene un efecto en las expectativas de los actores, que desarrollan procesos de aprendizaje a partir de las experiencias en arenas políticas similares a otros niveles. Por último, hemos ilustrado cómo en este tipo de política distributiva, la batalla por el poder en las instituciones de gestión es más determinante de

47. Si bien es cierto que los planes catalanes de investigación presentan elementos de confluencia con los planes nacionales de I+D. 
la orientación de la política que la opción por uno u otro instrumento de intervención.

Los resultados del análisis de la política de ciencia, tecnología e innovación catalana están en consonancia con las explicaciones que ponen énfasis en el peso de los grupos de interés en las políticas y en la formación de policy communities (Walker, 1991; Baumgartner y Leech, 1998) y en los efectos de refuerzo de las instituciones, incluso contra las preferencias reveladas de los gobiernos (Thelen y Steinmo, 1992). Como se ha dicho, el carácter preferentemente distributivo de la política la convierte en un terreno propicio para la intervención y presión de los intereses mejor organizados y con mayor necesidad de aquello que se distribuye. La mayoría de los actores de la investigación, especialmente los públicos, como universidades, centros de I+D o investigadores individuales, se caracterizan por una fuerte dependencia de los recursos para su financiación. Esta situación estructural hace que los intereses de los actores de la $\mathrm{I}+\mathrm{D}$ tiendan bien a movilizarse o bien a presentarse ante los diferentes gobiernos para conseguir que éstos les asignen recursos que cubran sus necesidades para la $\mathrm{I}+\mathrm{D}$. En cambio, los actores de investigación privados, en especial las empresas, no tienen esta dependencia de los recursos públicos y, por lo tanto, ni se movilizan ni presionan con tanta fuerza al gobierno regional.

El caso catalán nos demuestra, por último, las posibilidades de llegar a compromisos o a situaciones de consenso, en las cuales el discurso político de alto nivel se orienta hacia el mundo empresarial, hacia la creación de riqueza por medio de la innovación y, sin embargo, dada la estructura institucional que se ha creado, las actuaciones centrales se dirigen a favorecer los intereses académicos y tienen la orientación tradicional de las políticas de investigación. ${ }^{48}$

\section{Bibliografía}

BACARÍA, Jordi; BorRÁS, Susana; FERnÁNDEZ-Ribas, Andrea (2001). «El sistema de innovación regional en Cataluña». En OlazArán, Mikel; GÓmEZ URANGA, Mikel (eds.) (2001). Sistemas regionales de innovación. Bilbao: Servicio Editorial Universidad del País Vasco, p. 221-249.

BANCO Mundial (1999). El conocimiento al servicio del desarrollo. Informe sobre el desarrollo mundial. Madrid: Ediciones Mundi-prensa.

Baumgartner, Frank R.; LeECH, Beth L. (1998). Basic Interest. The importance of Groups in politics and in Political Science. Princeton: Princeton University Press. BraczycK, H-J.; COOKe, P.; HeINDERINCH, M. (eds.) (1998). Regional Innovation Systems. Londres: University College.

48. Este caso debe compararse con el giro radical de la política científica y tecnológica española que la llegada del Partido Popular ha supuesto en favor de una política de orientación empresarial, con la puesta en marcha de iniciativas de apoyo a las empresas para "corregir» la orientación excesivamente académica del periodo anterior (Sanz Menéndez, 1997). Por otro lado, las políticas del gobierno vasco de orientación tecnológico-industrial también son un elemento para comprender las diferencias (Moso y Olazarán, 2001). 
Caracostas, Parakevas; Muldur, Ugur (1998). Society, The endless frontier. A European vision of research and innovation policies for the $21^{\text {st }}$ century. Luxemburgo: European Communities. EUR 17655.

Carmines Edwars G.; Stimsom, James A. (1989). Issue Evolution. Princeton: Princeton University Press.

CoOke, P.; GÓmez, M. (1998). «Dimensiones de un sistema de innovación regional: organizaciones e instituciones». Ekonomiaz, 41, p. 46- 67.

CoOke, P.; Gómez-Uranga, M.; Etxebarría, G. (1997). «Regional innovation systems: Institutional and organizational dimensions». Research Policy, vol. 26, p. 475-491.

DURÁN, Alicia (coord.) (1999). La geografía de la innovación: ciencia, tecnología y territorio en España. Madrid: Los Libros de Catarata.

EC- European Commission (1995). Green Paper on Innovation. Bruselas: EC, COM (95) 688 (final).

EDQUIST, Charles (ed.) (1997). Systems of innovation. Technologies, institutions and Organisations. Londres: Pinter.

Fernández, María; SAnZ Menéndez, Luis; Cruz Castro, Laura (2003). «Diseño institucional y preferencias políticas: o cómo equilibrar los intereses académicos en la política de ciencia, tecnología e innovación gallega». Inguruak-Revista Vasca de Sociologia y Ciencia Politica, 35, abril, p. 33-68.

FreEMAN, Christopher (1995). "The "National System of Innovation" in Historical Perspective». Cambridge Journal of Economics, vol. 19, n. ${ }^{\circ}$ 1, febrero, p. 5-24.

GC-Generalitat de Catalunya (1993). Pla de Recerca de Catalunya (1993-1996).

Barcelona: CIRIT-Comissionat per a Universitats i Recerca.

- (1997). II Pla de Recerca de Catalunya (1997-2000). Barcelona: CIRIT-Comissionat per a Universitats i Recerca.

- (2000). El sistema universitari de Catalunya. Memoria del curs 1998-1999. Barcelona: Generalitat de Catalunya.

- (2001a). III Pla de Recerca de Catalunya (2001-2004). Barcelona: DURSI-CIRIT.

- (2001b). Pla d'Innovació de Catalunya (2001-2004). Barcelona: CIDEM-DICT.

GonzÁlez de la FÉ, Teresa (2001). "Las Políticas regionales de Ciencia, Tecnología e Innovación. Análisis Comparativo». V Congreso Español de Ciencia Política y de la Administración, La Laguna-Tenerife, 26-28 de septiembre de 2001 (mimeo). Hall, Peter A. (1989). "Conclusion: The Politics of Keynesian ideas». En Hall, Peter A. (ed.). The Political Power of Economic Ideas. Keynesianism across Nations. Princeton (NJ): Princeton University Press, p. 361-391.

- (1993). «Policy paradigms, social Learning, and the State». Comparative Politics, 25, p. 275-296.

Hedström, Peter (1998). "Rational imitation». En Hedström, Peter; SwedberG, Richard (eds.) (1998). Social mechanism. An Analytical Approach to Social Theory. Cambridge: Cambridge University Press, p. 306-327.

KIngDON John W. (1984/1995). Agendas, Alternatives and Public Policies. HarperCollins ( $2^{\mathrm{a}}$ ed.).

LowI, Theodore. J. (1972). «Four systems of policy, politics and choice». Public Administration, vol. XXXII, n.o 4, julio-agosto, p. 298-310.

LundVAlL, Ben Ake (ed.) (1992). National systems of innovation. Towards a Theory of innovation and Interactive Learning. Londres: Pinter.

Maltrás, Bruno; Quintanilla, Miguel A. (1992). Producción cientifica española 1981-1989. Madrid: CSIC. 
MARTín, Carmela; Moreno, Lourdes; ROMERO, Luis R. (1990). «Actividades tecnológicas y estructura productiva regional». ES. Economía y Sociedad, n. ${ }^{\circ} 4$, diciembre, p. 119-136.

Metcalfe, S.E.; Georghiou, Luke (1998). «Equilibrium and Evolutionary Foundations of Technology Policy». STI Science, Technology and Industry Review, n. ${ }^{\circ} 22$, p. $75-100$.

Moso, Mónica; OlazArán, Mikel (2001). «Actores, ideas e instituciones: políticas tecnológicas regionales y creación de un sistema de $\mathrm{I}+\mathrm{D}$ en la Comunidad Autónoma del País Vasco». En Olazarán, Mikel; GÓmez Uranga, Mikel (eds.) (2001). Sistemas regionales de Innovación. Bilbao: Servicio Editorial Universidad del País Vasco, p. 406-432.

NeLson, Richard (ed.) (1993). National Innovation Systems. A Comparative Analysis. Oxford: University Press.

Nelson, Richard R.; ROMER, Paul M. (1996). «Science, Economic Growth, and Public Policy». En Smith, Bruse L.R.; Barfield, Claude E. (eds.) (1996). Technology, $R \& D$ and the Economy. Washington DC: The Brookings Institution-American Entreprise Institute, p. 49-74.

Olazarán, Mikel; Gómez Uranga, Mikel (eds.) (2001). Sistemas regionales de Innovación. Bilbao: Servicio Editorial Universidad del País Vasco.

Peterson, John; SHARp, Margaret (1998). Technology Policy in the European Union. Londres: MacMillan.

PUIG SAlELLAS, Josep M. (1991). «El marc legal de la recerca». CIRIT, La recerca a Catalunya, repte de futur. Barcelona: Generalitat de Catalunya, p. 5-20.

RibA, Marta; LEYDESDORFF, Loet (2001). «Why Catalonia cannot be considered as a Regional Innovation System?». Scientometrics, 50, p. 215-240.

Romero, Marta; CruZ, Laura; SANZ MenÉNDEZ, Luis (2003). «Estabilidad y cambio en las políticas andaluzas de ciencia, tecnología e innovación». Revista Internacional de Sociología, 35, mayo-agosto, p. 7-52.

SANZ MenÉNDEZ, Luis (1997). Estado, ciencia y tecnología en España: 1939-1997. Madrid: Alianza Universidad.

- (2001). «¿Por qué cambian las políticas?: La política europea de investigación y desarrollo tecnológico». Revista Española de Ciencia Política, 4, abril, p. 97-121.

SANZ MENÉNDEZ, Luis; ARIAS, Esther (1998). «Concentración y especialización regional de las capacidades tecnológicas: un análisis a través de las patentes europeas». Economía Industrial, 324, p. 105-122.

Sanz Menéndez, Luis; Cruz Castro, Laura; Romero de la Cruz, Marta (2001). «Recursos, intereses y difusion de modelos para la política regional de I+D: La Comunidad de Madrid». En Olazarán, Mikel; Gómez Uranga, Mikel (eds.) (2001). Sistemas regionales de innovación. Bilbao: Servicio Editorial Universidad del País Vasco, p. 375-403.

SCHÖN, D.A.; REIN, M. (1994). Frame reflection. Towards the resolution of intractable policy controversies. Nueva York: Basic Books.

SKOCPOL, Theda; FINEGOLD, K. (1982). «State capacity and economic intervention in the early New Deal». Political Science Quarterly, vol. 97, n. ${ }^{\circ}$ 2, p. 255-278.

SOler Llebaria, Josep (1997). «Las elecciones autonómicas en Cataluña (1980-1995)». En AlCÁNTARA, Manuel; MARTínEZ, Antonia (eds.). Las elecciones autonómicas en España, 1980-1997. Madrid: CIS.

Thelen, K.; Steinmo, S. (1992). «Historical Institutionalism in Comparative Analysis». En Steinmo, S.; Thelen, K.; LONGSTRETH, F. (eds.) (1992). Structuring 
Politics. Historical Institutionalism in Comparative Analysis. Cambridge: CUP, p. 1-32.

VILALTA, Josep M. (2000). «Polítiques de Ciència i Tecnologia a Catalunya». En GOMÁ, Ricard; SubiraTs, Joan (ed.) (2000). Govern i Polítiques Públiques en Catalunya 1980-2000. Barcelona: Edicions Universitat de Barcelona- Bellaterra: Universitat Autònoma de Barcelona, p. 61-84.

WalKer, Jack L. Jr. (1991). Mobilizing interest groups in America. Ann Arbor: The University of Michigan Press.

ZIEGLER, J. Nicholas (1997). Governing Ideas. Strategies for Innovation in France and Germany. Ithaca: Cornell University Press. 